\title{
Industrialization of a Large Advanced Ultrasonic Flexible Probe for Non-destructive Testing of Austenitic Steel Pieces with Irregular Surface
}

Frédéric Lasserre ${ }^{1}$, David Roue ${ }^{2}$, Juergen Laube ${ }^{3}$, Philippe Dumas ${ }^{4}$, Bruno Kingler ${ }^{4}$, François Cartier ${ }^{2}$ and Olivier Roy $^{5}$

1. Technical Engineering Department, AREVA Nuclear Power, Paris 94300, France;

2. CEA LIST Department, CEA SACLAY, Gif sur Yvette, 91191, France;

3. IBGSI Department, AREVA GmbH, Erlangen 91052, Germany;

4. IMASONIC, Voray-sur-l'Ognon, 70190, France;

5. M2M, Les Ulis, 91940, France

\begin{abstract}
Because the UT (ultrasonic testing) flexible probe technology may be an appropriate answer to examine components with uneven surface, AREVA has developed an industrial application of the CEA's (French Atomic Energy and Alternative Energies) flexible phased arrays sensors. As a "first of a kind" project, the challenges faced were significant, including developing a phased array smart probe suitable for industrial use on rather simple but large scale geometries, permitting UT propagation within a constraining media structure and then targeting a qualification according to ENIQ (European Network for Inspection Qualification) methodology. A prototype flexible probe, designed for UT validation, and final flexible linear array probes permitting the UT behavior (as, e.g., detection and sizing from diffraction type echoes) to be maintained on wavy coupling surfaces, have been manufactured. These probes include a profilemeter with optical sensors control and a specifically designed coupling circuit (avoiding probe housing tightness issues). Qualification has been performed using open test blocks, (where known "defects" exist, for procedure qualification), and blind test blocks, (where "defects" are unknown, for qualification of testing personnel). One open test bloc was customized to represent a "real" surface condition, with gaps up to $2.5 \mathrm{~mm}$ under the regular rigid probes. AREVAI/BGSI in Germany was selected to lead the project, with assistance in development and manufacturing sub-contracted to "CEA/LIST" laboratory, and the companies "IMASONIC" and "M2M". This paper describes the development of these probes and explains a few features (ENIQ qualification objectives fulfilled, UT data acquired on actual perturbed surface) that made their industrial implementation successful.
\end{abstract}

Key words: Ultrasonic testing, phased arrays, flexible probe technology, uneven surface.

\section{Introduction}

\subsection{Background}

The nuclear industry features often heavy components that are subject to stringent safety regulations. For example, the primary components in the reactor building area are composed of many tons of steel manufactured, welded, installed and safety

Corresponding author: Frédéric Lasserre, Ph.D.; research fields: physical acoustic, and ultrasonic testing. E-mail: frederic.lasserre@areva.com. classified to the highest safety level, thus requiring frequent non-destructive examinations to verify their $100 \%$ conformity.

Some of the components include austenitic steel welds which require end of manufacturing as well as later in-service validations by means of non-destructive tests or examination, ("NDT" or "NDE"). Ultrasonic testing ("UT") is thus often used. However, the criteria required for its successful application are often challenging as a result of the size and nature of the assemblies to be tested and the 
required sensitivity levels.

Whilst use of NDT methods such as radiographic testing can be problematic due, for instance, to its sensitivity to thickness variations, (thus making the required density homogeneity of the resulting image difficult to obtain), UT on the other hand is sensitive to metal "structure" and surface unevenness which can prevent sufficient probe coupling and cause sound field disturbances preventing successful use of contact UT techniques.

Besides, in specific cases of large austenitic butt welds, AREVA, (as a provider of nuclear island components), often needs to examine uneven surfaces, for example, that result from pipe bending, but such uneven scanning surfaces have also an impact on sound propagation by modifying the sound field parameters inside the structure that renders the use of UT-less reliable.

Such a type of surface state was however identified as being within the scope of application of the flexible probe technology developed by the CEA (French Atomic Energy and Alternative Energies) and in practical application for several years.

In order to have an available and reliable industrial NDE tool for such cases, a qualification exercise was launched to develop and implement a smart contact flexible phased array probe.

A first UT feasibility study was performed followed by the specification of the probe in its final operating environment. Appropriate UT system features and the mechanical means required to hold and control the probes were then developed in parallel.

The final adapted design of the completed NDE equipment thus corresponded to the geometry and the structure of the components under consideration. The equipment including the flexible probe is intended for use locally on the surface irregularities, as a "back-up" solution when the limits of regular UT phased array technology are exceeded.

This equipment is currently undergoing qualification validation phase which is presented in this paper, together with the first results demonstrating the capabilities of the test blocks.

\subsection{Flexible Probe Technology}

The flexible probe technology developments started almost 20 years ago (see example in Ref. [1]). The CEA had started working on it in the early 2000's, (see Ref. [2]), and since then, several industrial applications [3] have been identified.

The challenge here was to perform a UT examination on an irregular (not flat), surface profile such as with the components introduced above, using phased array contact probes with a flexible probe wear plate.

Fig. 1 describes the principle of the so-called "smart"

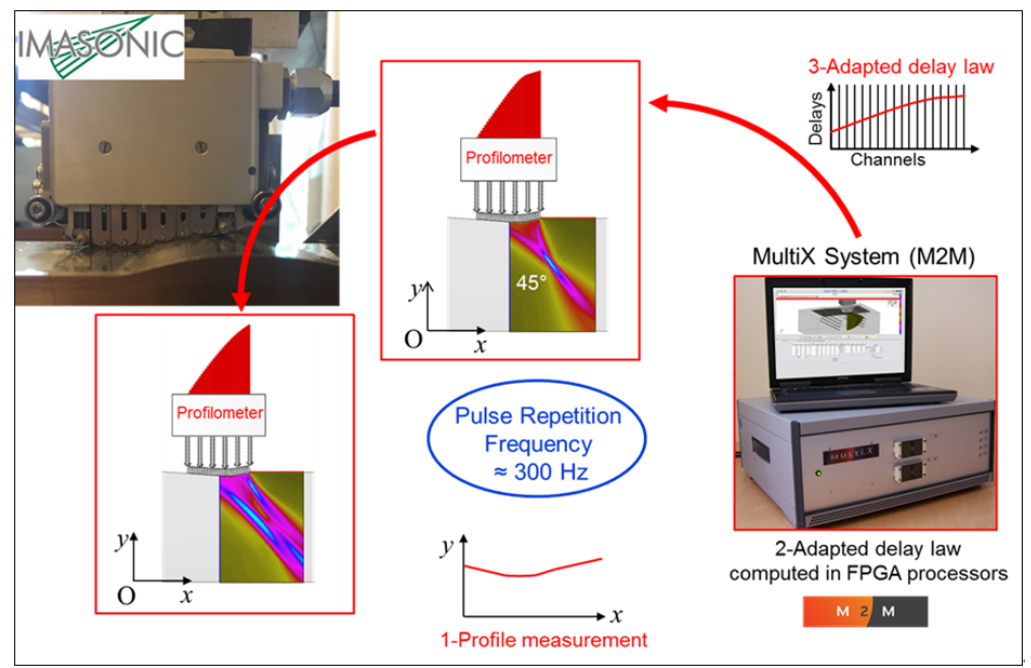

Fig. 1 Smart flexible phased array principle. 
flexible phased array with the different steps performed in real-time (faster than the usable pulse repetition frequency) by:

(1) Profile measurement of the irregular surface with the profilemeter;

(2) Adapted "surface-reverse" delays computed in electronic device;

(3) Applied new delay laws adapted to the surface contours.

The profilemeter system is based on optical measurements.

Under this system, the profile of a complex surface is reproduced on the flexible array and the related deformation is determined by the optical measurement of the vertical displacement of the pistons (see Fig. 2). Then, the displacement of each element is interpolated using an appropriate interpolation curve (using "spline cubic" interpolation). Both the interpolation and delay law computation are performed in line with the UT system real time processing.

With the appropriate new surface related delay laws applied to the UT beams, the resulting examination data is then recorded.

\section{Specifications}

Many actual outer profiles were collected at the start on pipework with circumferential austenitic butt narrow gap welds in order to define the parameters for the design of the flexible array probe. The profiles were "finger-printed" all around the parts in the vicinity of the weld center lines and recorded.
This allowed the range of variation and thus the scope of potential interest of the tool to be estimated.

The profiles taken into consideration vary progressively and smoothly, even on large areas, with no circumferential gap expected, even after taking into consideration the natural curvature of the outer diameter.

The development of an appropriate flexible probe design was thus launched on a "one dimension" ("1D", linear array), probe type basis.

Following completion of the feasibility study, the physical characteristics of the new flexible probe were defined starting from those of the existing prototype probe that had been used so far. The goal was to increase the sensitivity of the probe.

The probe frequency was fixed at $1.5 \mathrm{MHz}$. This value is coherent with the size of the targeted defects and limits so far as possible the attenuation phenomenon (attenuation being proportional to (frequency) ${ }^{2}$ ).

These specifications were confirmed as appropriate during the UT pre-qualification trials performed with the manufactured prototype 48 element probe.

The following step, being specification of the final probe, aimed at taking into account:

- the results from the test blocks with the prototype probe;

- the UT acquisition and analysis software and hardware;

- the manipulator, for automated examination on the pipe welds;

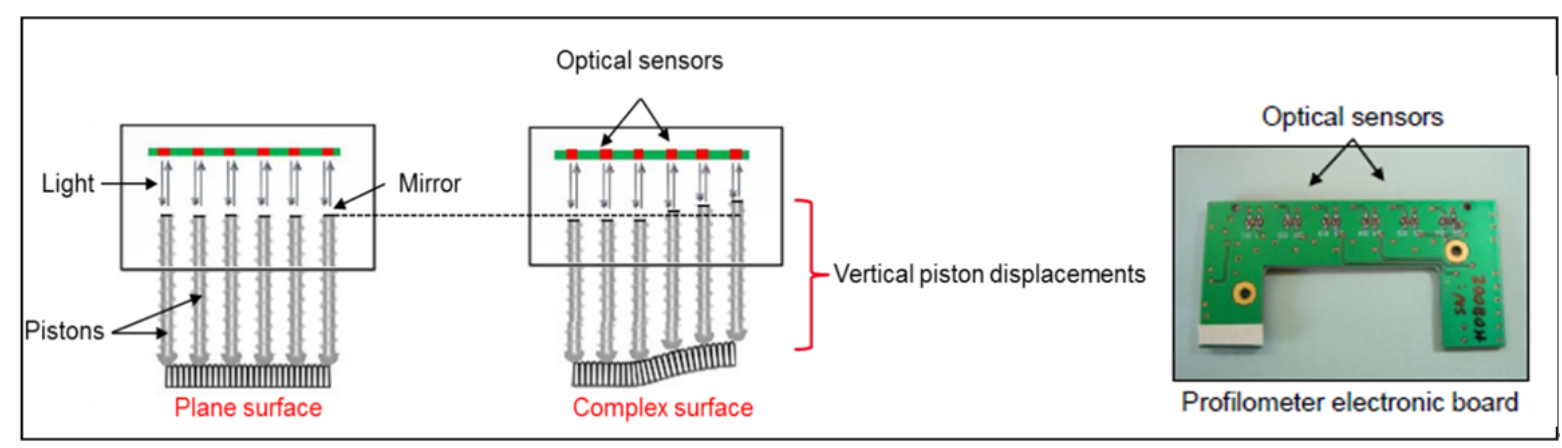

Fig. 2 Optical measurement of the aperture deformation. 
Table 1 Specifications for the final probes.

\begin{tabular}{ll}
\hline Specification & Value \\
\hline Usable linear range of the profilemeter (maximum stroke of the pistons for optical measurement) & $30 \mathrm{~mm} *$ \\
Tightness against coupling media & Satisfactory \\
Cleaning optical measurement area & Available \\
Blocked rotation piston (increase of accuracy for OD profile measurement) & $\pm 2.5^{\circ}$ \\
\hline
\end{tabular}

*: a maximum mechanical stroke displacement of $40 \mathrm{~mm}$.

- the profilemeter, to be able to handle the maximum specified surface irregularities to be considered;

- the adaptations directly linked to the in-situ configuration, and found necessary to be made during the dedicated trials on the test blocks;

- specific feasibility studies from the manufacturer.

The main challenges handled during the final specification are summarized in the table of Table 1.

Pictures representing the final probe are given in the following section.

\section{Manufacturing}

Probes have been manufactured by IMASONIC company under license from the CEA. Compared to a "conventional" phased array probe, flexible arrays are very specific for the following reasons:

- The probe is made of many individual elements, each of which is considered as an independent transducer and must integrate a "matching" layer, a piezo composite material, as well as a damping function, and all this in a very compact volume;

- A complex structure must be implemented inside the probe; this is a mechanical system made of pistons, allowing matching of the flexible part on the inspected surface, and the measurement profile system comprising opto-electronic components;

- In addition, the final probes designed for this application integrated two dedicated new specificities:

(1) an active aperture that is the largest active part IMASONIC has ever manufactured for a "1D" flexible array (which involved changing the electro-acoustical design for a significantly different one, and adapting each individual casing to its new dimensions);
(2) a new profile measurement system with a larger stroke, integrating new components.

The total active length of the final probes is about $70 \mathrm{~mm}$. The frequency is $1.5 \mathrm{MHz}$ and there are 48 elements in a one dimension array arrangement (for both prototype and final probes).

The pistons have a maximum mechanical stroke between $40 \mathrm{~mm}$ and $50 \mathrm{~mm}$.

The profilemeter uses optical detection of the displacement of the pistons. Accuracy of the system was improved by limiting the rotation of each piston during their linear displacements.

These requirements make this type of probe (refer Fig. 3), unique compared to typical UT probes (refer Fig. 4).

IMASONIC performed thousands of compression cycles of the probe pistons to test their durability and to verify the impermeability of the probe casing in relation to the coupling medium, the probe being intended to be usable in all positions and orientations.

The resulting new manufactured optical measurement system is able to operate in situations with a height displacement of more than $35 \mathrm{~mm}$, thus meeting the one of the initial principal requirements (refer above to "specifications").

\section{Equipment}

\subsection{UT System}

The UT system used for this application is a phased electronic system with 64 channels in parallel manufactured by M2M company ("MULTIX++"). $\mathrm{M} 2 \mathrm{M}$, in collaboration with the CEA, needed to develop a new software version in order to improve the performance of the data acquisitions (including 


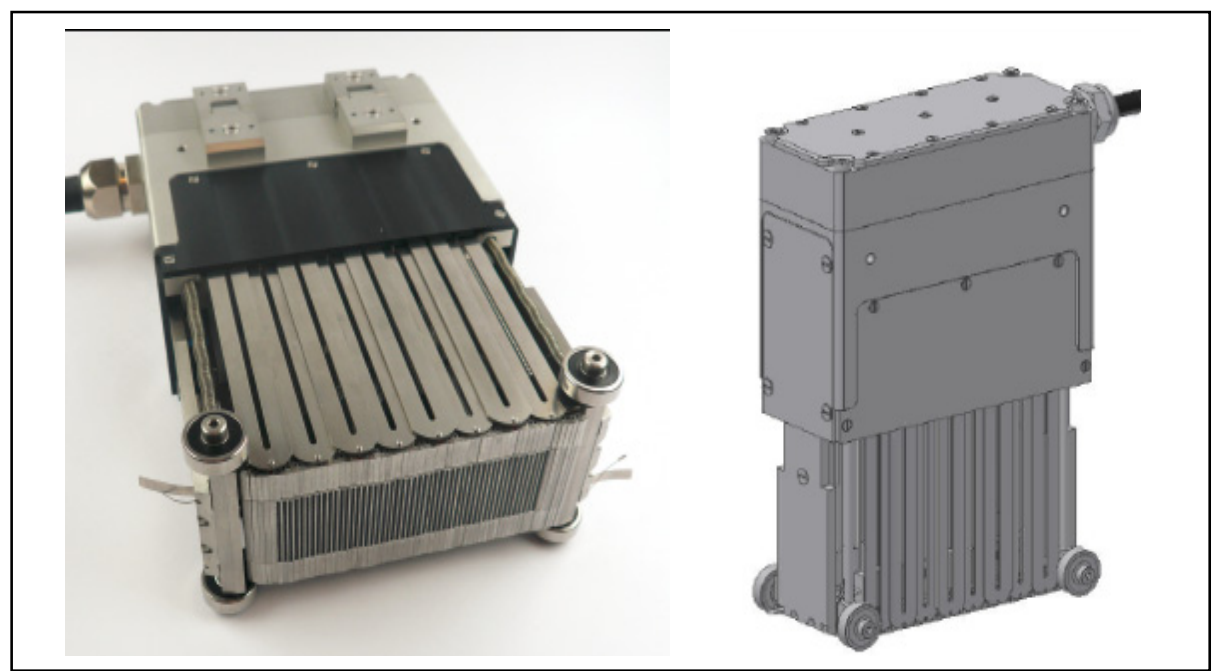

Fig. 3 Final linear flexible probe applicable to large surface variations.

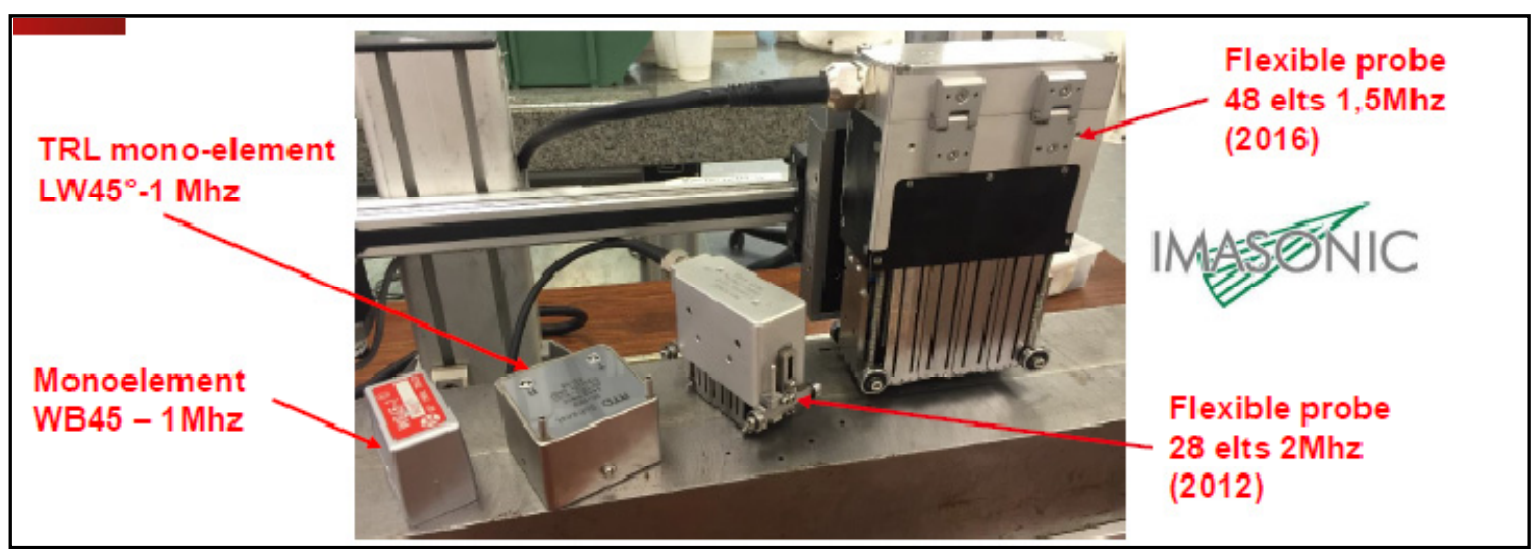

Fig. 4 Final linear flexible probe size compared to other types of UT probes.

unlimited shots, several salvo (or "acquisition cycles"), and optimization of specific data from the profilemeter).

Some specific options devoted to use of profilemeter measurements have also been developed:

- a "clear" button to adjust the reference altitude;

- an instrument alarm warning when the profilemeter is out of range (blue button in Fig. 5), in the parameter and acquisition panel;

- a mechanical CSCAN ("top view"), alarm in acquisition panel.

The data analysis is done with a new version of CIVA software (multi-technique platform for data analysis). It takes into account the access CSCAN alarm view and new specific tools.

The software enables in line checking of the quality of the acquisition. In addition, the profile measured by the optical system is included in the true BSCAN view ("side view").

\subsection{Mechanics}

Use of the flexible probe is done with the same basic robotics arrangement already in place for regular UT inspection undertaken with standard phased array probes: typically a circumferential track and a 2-axis robot and control system (see Fig. 6). The main adaptations to the robotic system are:

- coupling system utilizing a special gel mix instead of water to reduce the risk of infiltration of the coupling medium into the flexible probe sensor;

- reinforcement and optimization of the probe holder to accommodate the increased weight of the 


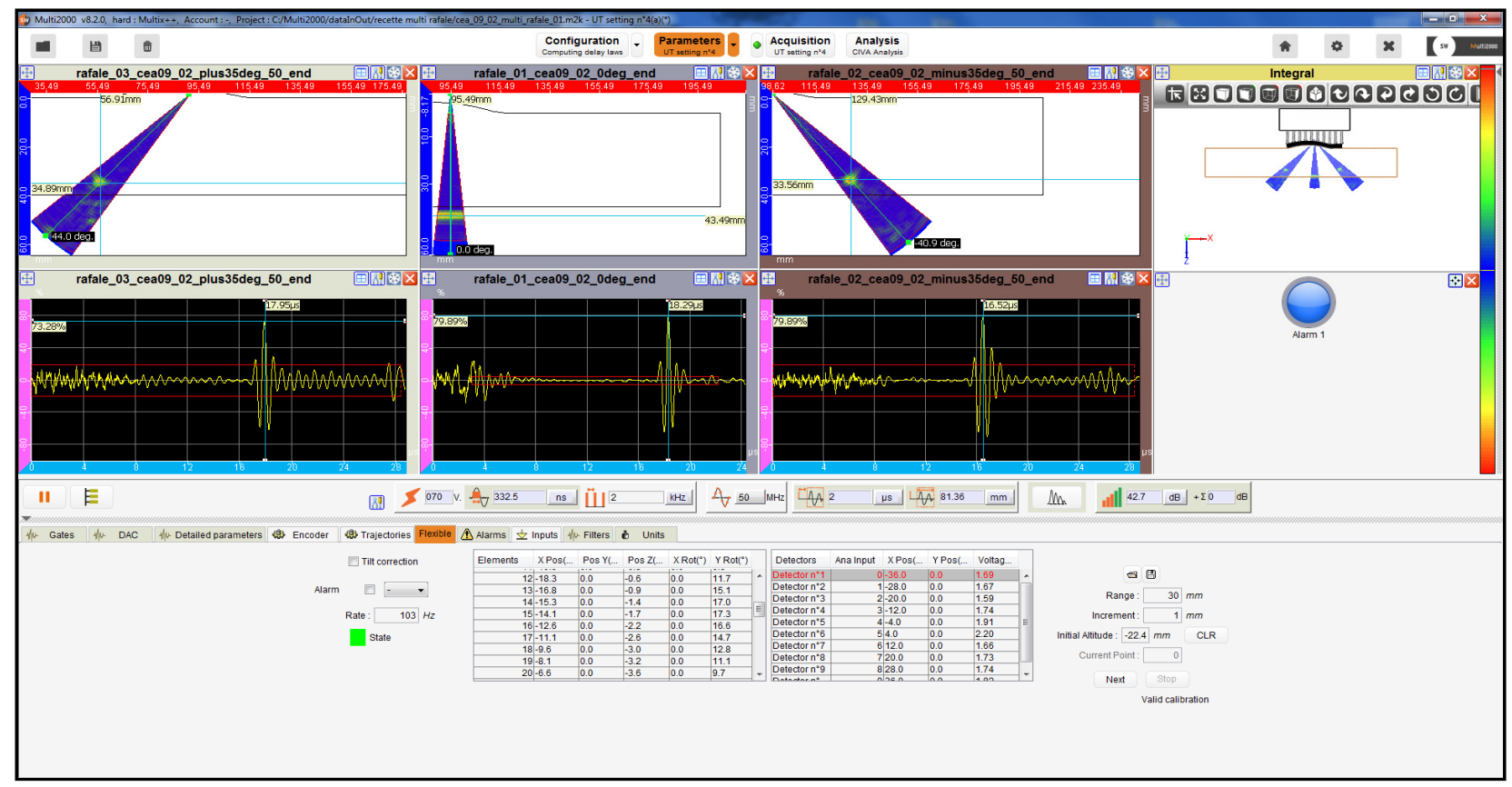

Fig. 5 Example of set-up display screen with three salvos and information about the profilemeter of the flexible probe.

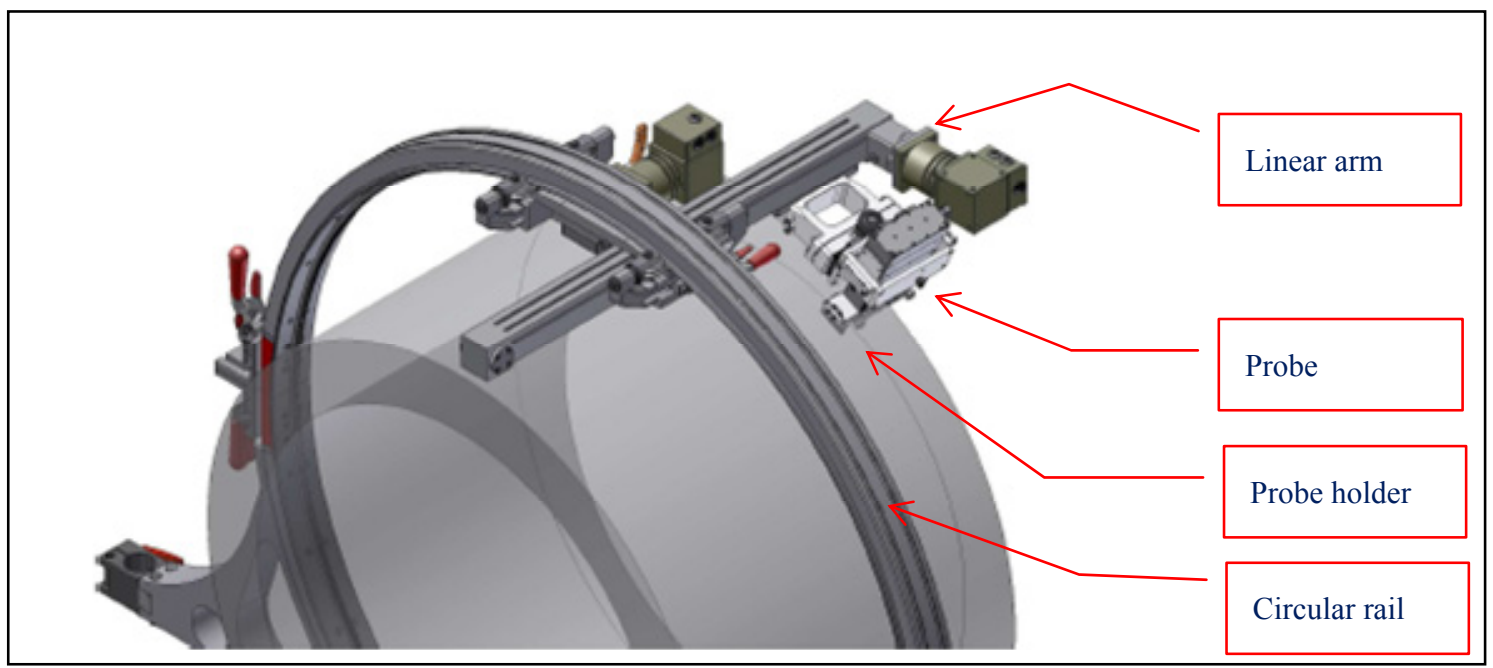

Fig. 6 3D CAD (computer aided design) view of the robotic.

probe, and support to ensure the main probe axis remain parallel to the inspection surface (see Fig. 7).

\section{Qualification}

\subsection{Outline}

The qualification process followed is based on ENIQ (European Network for Inspection Qualification) recommendations. The principles of ENIQ qualification are to first demonstrate that the intended equipment fulfils the pre-defined inspection objectives, and secondly that the NDT operators can detect and characterize flaws during "blind tests" with this same equipment.

All details are set out in a comprehensive qualification procedure which encompasses use of the inspection equipment and procedure.

Qualification is demonstrated by use of open tests, whilst the personnel qualification is based on certification and internal qualification obtained during the blind tests. 


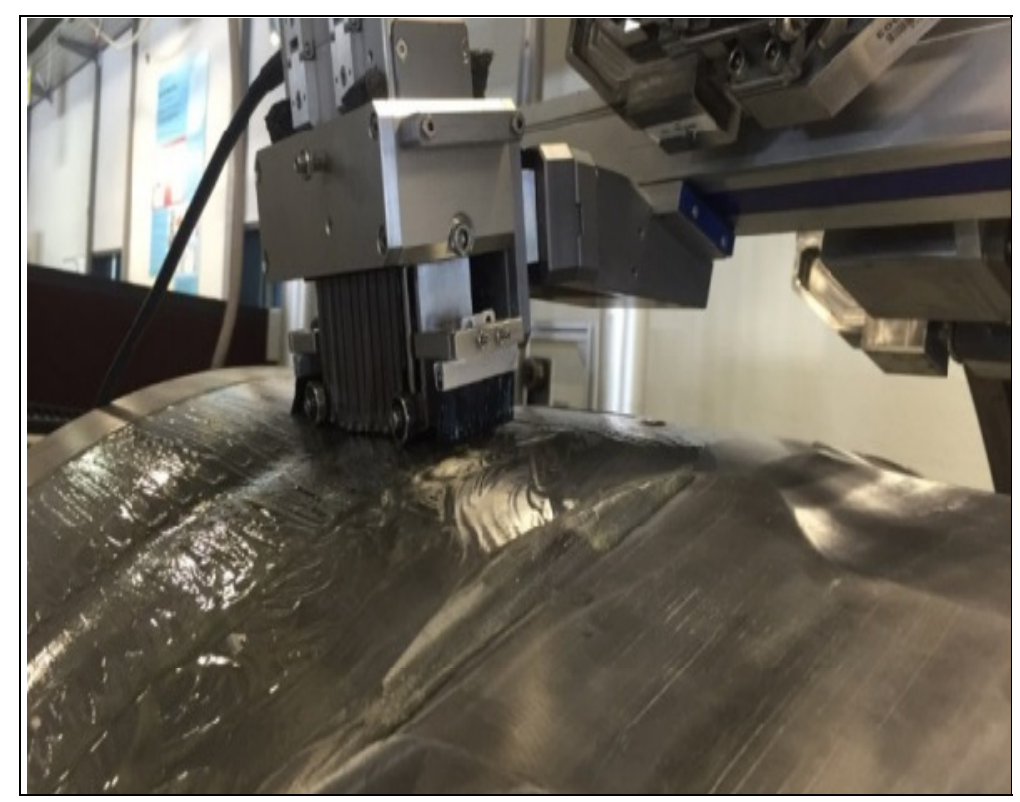

Fig. 7 Final linear flexible probe in situation and its probe holder.

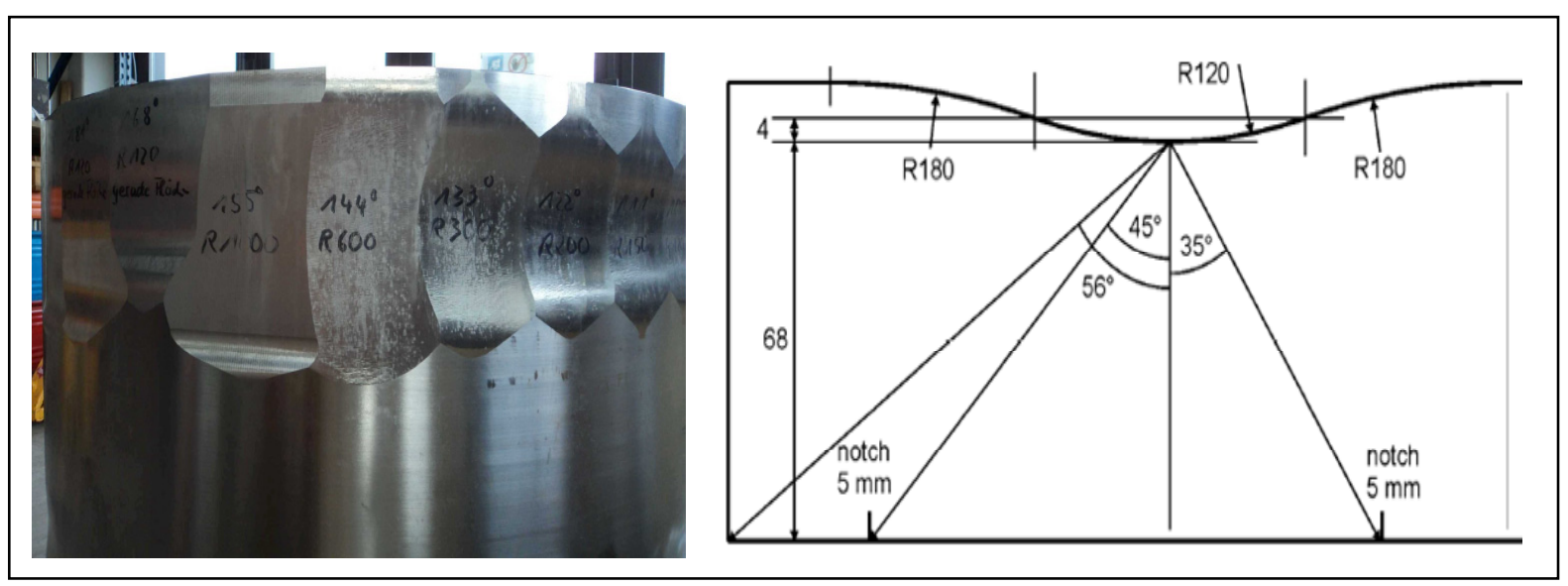

(a)

(b)

Fig. 8 Qualification block with calibrated unevenness surface: (a) worse case of gap curvature; and (b) location of notches at the detection impingements.

Qualification files have already been prepared for the inspection of the same components using standard phased arrays technology and as well as with the flexible probe, using the same test blocks (i.e., those with regular surfaces only).

The results obtained with the flexible probe equipment are thus complementary and permit a direct comparison.

\subsection{Test Blocks}

Several test blocks were used during the feasibility and qualification stage, all of which were in austenitic material:

- One flat block with calibrated curved gaps for a basic initial checking of the flexible technology capabilities;

- Two blocks with the same geometry as the parts, containing realistic and artificial flaws, one block had the same geometry as the parts to be examined, with locally border line anisotropic structure, with an angular sector containing notches and machined external profiles (see Fig. 8a). Different gap heights are simulated on the block (see the scheme for the worse case in Fig. 8b). The notches at the opposite 
wall have been located so that the detection positions for the flexible probe correspond exactly to the gaps locations when detection is obtained in the refraction angle domain range $\left(35^{\circ}-56^{\circ}\right)$.

All the blocks had a metallographic structures representative of actual parts.

\subsection{Process}

The first part of the qualification was performed with the prototype 48 element probe (shown during tests in Fig. 9).

Phased arrays beam in $-60^{\circ}$ to $+60^{\circ}$ angle range were used, with optimized point focusing.

With the prototype probe, when not implementing the final version of the profilemeter, the results in detection and sizing of the defects on the qualification block with even surface (gap $<0.5 \mathrm{~mm}$ ), are at least as good as with the regular rigid phased array probes.

On uneven surface (gap up to $\sim 2.5 \mathrm{~mm}$ under a 50 $\mathrm{mm}$ rigid probe), where some defects could not be detected with the regular phased array probes, using the flexible probe, the detection of diffraction type echoes was achieved with an SNR (signal-to-noise ratio) of at least $6 \mathrm{~dB}$, as illustrated in Fig. 10.

In this figure, the corner echo and the upper tip diffraction echoes are clearly exhibited despite the disturbance during the scanning. The effective propagation mode displayed here is $45^{\circ}$ compression wave.

The height sizing of the defects (notches and mechanical fatigue cracks) in the open test blocks was within $1.5 \mathrm{~mm}$ of true height.

The qualification open and blind tests have been completed using the final version of the industrial probe, which incorporated all the specifications indicated in Table 1.

Fig. 11 shows one of the screen displays for analysis during the reception tests (the display of merge data for several direction of propagation is also available).

Results obtained with the prototype probe could be utilized in addition since the acoustical part of the final probe is identical to the prototype with 48 elements.

The qualification process with the final flexible probe also included personnel training, inspection procedure and issue of the technical justification. Personnel were trained on the equipment, with confirmation of competency, before they performed the blind tests.

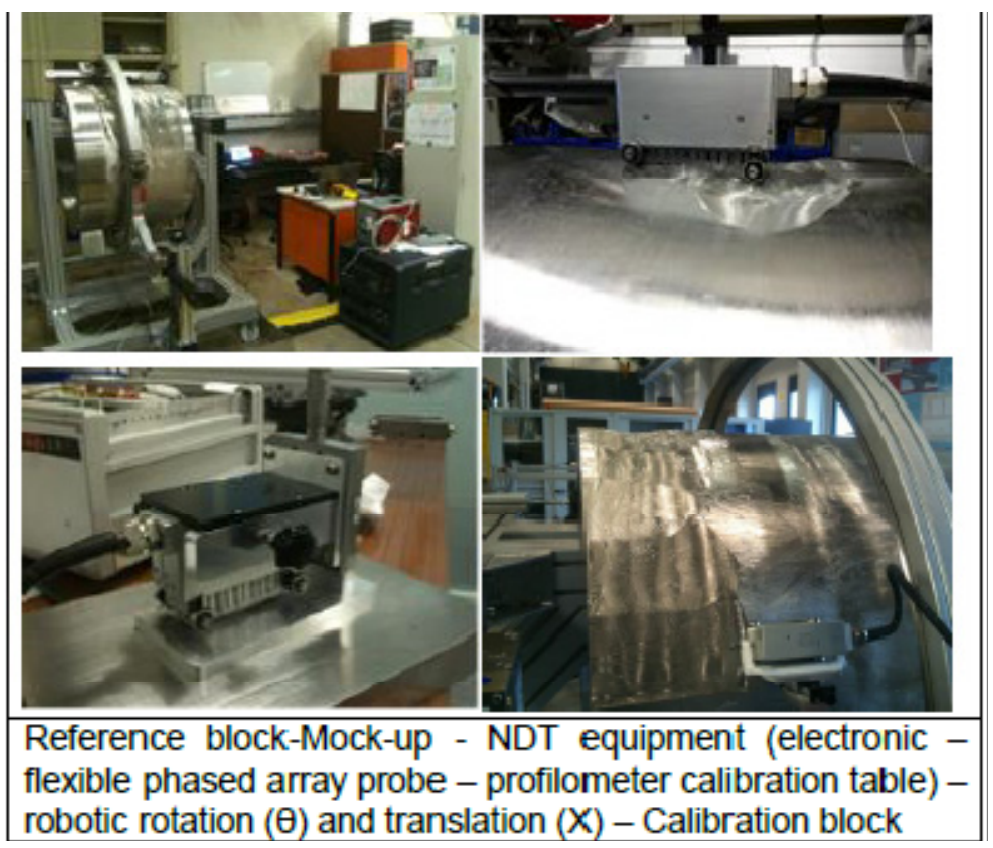

Fig. 9 Prototype flexible probe on the qualification block with artificial uneven surface. 
Industrialization of a Large Advanced Ultrasonic Flexible Probe for Non-destructive Testing of Austenitic Steel Pieces with Irregular Surface

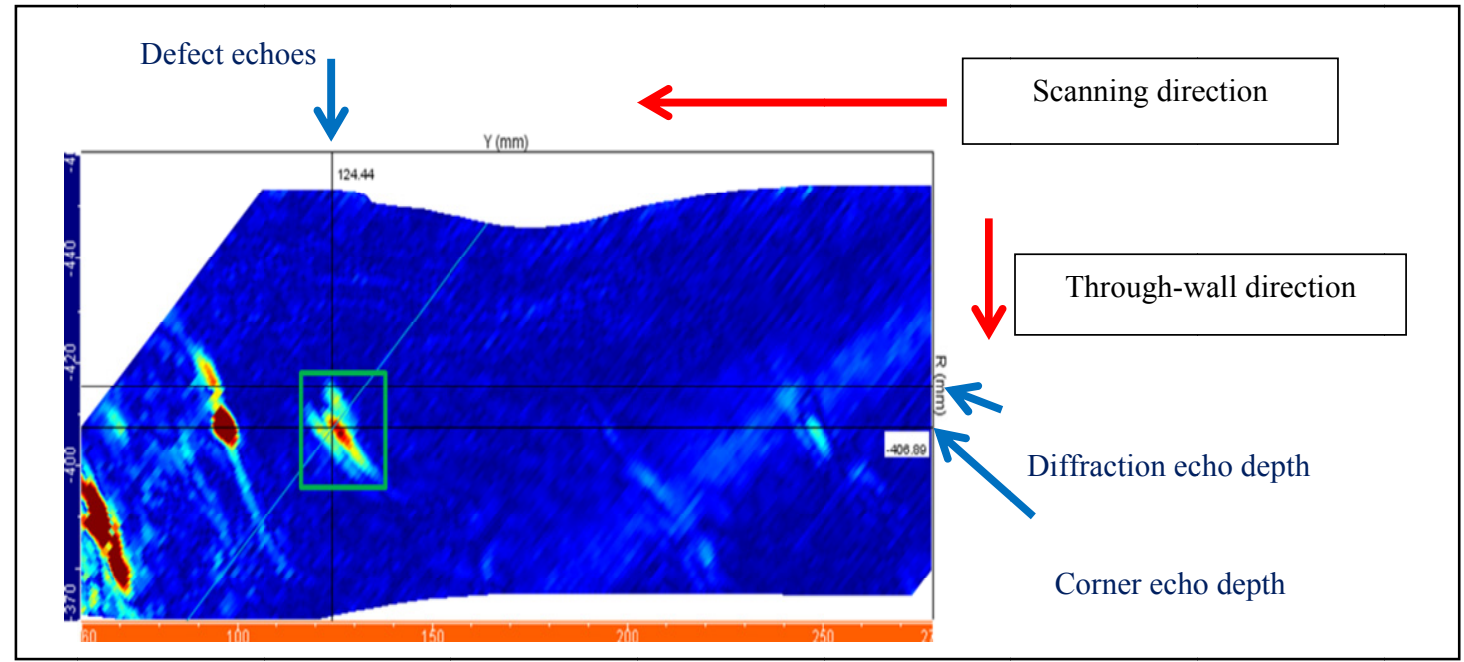

Fig. 10 BSCAN display of the 48 elements prototype probe scanning the gap block.

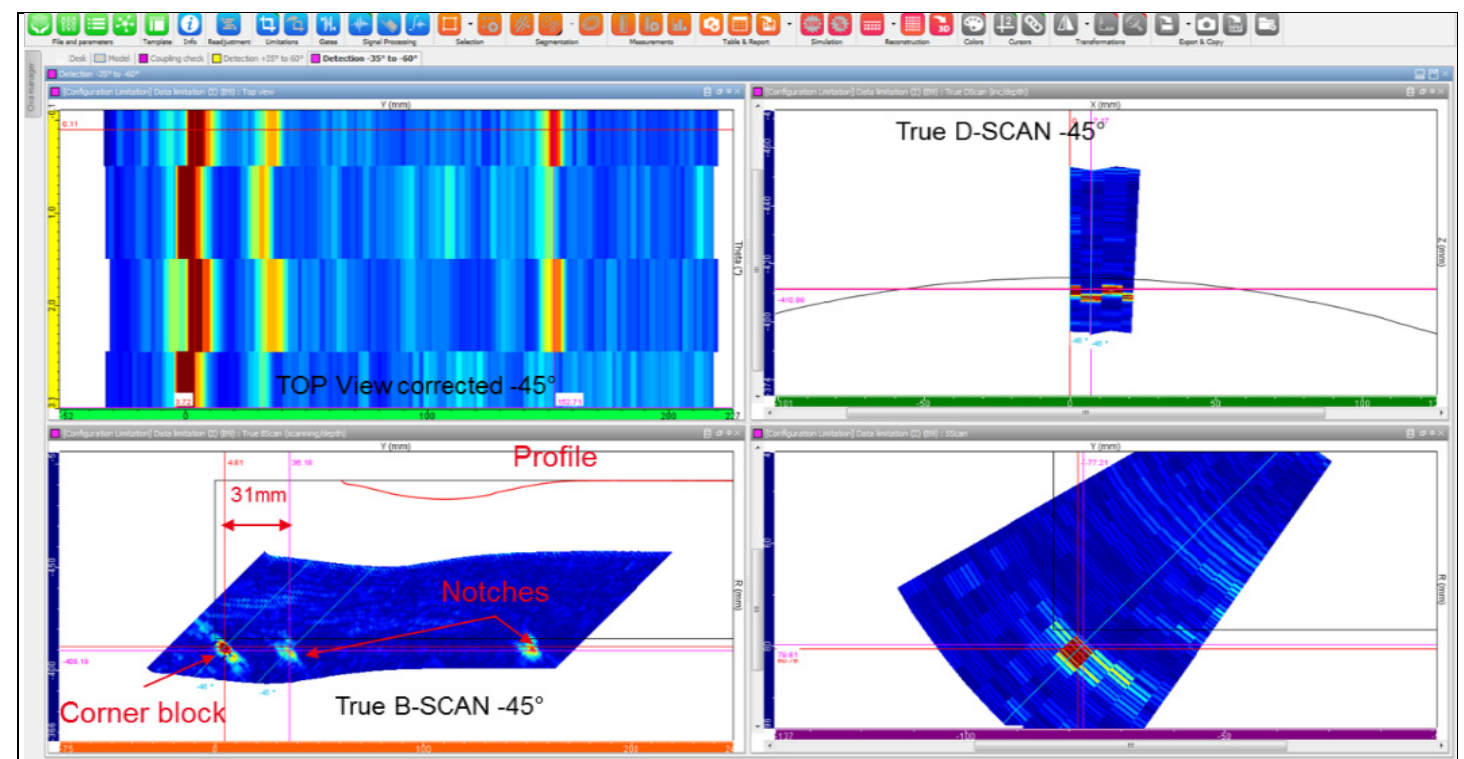

Fig. 11 Analysis display of one salvo from final probe in the worst case of gap curvature with final probe

\section{Conclusion}

The considerations made during the design phase and the outcome of the incorporated adaptations necessary for an industrial implementation of the flexible phased arrays technology have been presented in this paper.

AREVA presents this "first of a kind" method of examining austenitic material by UT when the surface is uneven, specifically out of the standardized gap range of $0.5 \mathrm{~mm}$ permitted for regular testing.

The project required a coherent and effective association of vendor, developer, and probe and UT system manufacturers.

At the start, AREVA had considered several potential solutions to handle the examination of irregular surface parts, and had made a technical comparison combined with a risk analysis to determine the preferred solution. This method, using flexible probe technology, has now proven itself to be capable of maintaining on uneven surfaces (within the pre-determined limits) at least equivalent capabilities as those that can be obtained using standard automated equipment on even surfaces. 
During its development, numerous challenges were overcome concerning the manufacturing of flexible arrays for such large vertical displacements, including the appropriately reinforced mechanics necessary for site implementation, and the use of an enhanced coupling medium to control coupling medium flow during use.

The final probe and the complete equipment have passed the end of manufacturing reception tests, the final "ENIQ" type qualification tests and dedicated in-service industrial implementation.

For the CEA, this project has been a good example of the industrial application of their patented flexible technology.

For IMASONIC and M2M companies, it has provided recognition of their advanced knowledge with a combination of a challenging target use, and a level of preparation for industrial implementation not previously achieved in the field of flexible probe technology.

For AREVA and its subsidiary IBGSI in Germany, the project has delivered a tool capable of meeting customers' needs regarding nuclear service inspection issues in as-built surface conditions, with the potential of being extended to other industrial fields.

\section{References}

[1] Powel, D. J., and Hayard, G. 1996. "Flexible Ultrasonic Transducer Arrays for Non-destructive Evaluation Applications." IEEE Trans. Ultrasonic, Ferroelectrics and Frequency Control 43 (3): 385-402.

[2] Mahaut, S., Roy, O., Casula, O., and Cattiaux, G. 2002. "Pipe Inspection Using UT Smart flexible Transducer." In Proceedings of the 8th ECNDT.

[3] Moussebois, D., and Casula, O. 2012. "Inspection of Complex 3-D Surfaces with Conformable UT PA Transducer." In Proceedings of the 9th ICNDE. 